\title{
Universiteit
}

Leiden

The Netherlands

\section{Natural deep eutectic solvents in plants and plant cells: In vitro evidence for their possible functions} Dai, Y.; Varypataki, E.M.; Golovina, E.A.; Jiskoot, W.; Witkamp, G.J.; Choi, Y.H.; Verpoorte R.

\section{Citation}

Dai, Y., Varypataki, E. M., Golovina, E. A., Jiskoot, W., Witkamp, G. -J., \& Choi, Y. H. (2021). Natural deep eutectic solvents in plants and plant cells: In vitro evidence for their possible functions. Advances In Botanical Research, 97, 159-184. doi:10.1016/bs.abr.2020.09.012

Version: $\quad$ Publisher's Version

License: $\quad$ Licensed under Article 25fa Copyright Act/Law (Amendment Taverne)

Downloaded from: https://hdl.handle.net/1887/3248724

Note: To cite this publication please use the final published version (if applicable). 


\title{
Natural deep eutectic solvents in plants and plant cells: In vitro evidence for their possible functions
}

\author{
Yuntao Dai ${ }^{\mathrm{a}, *}$, Eleni Maria Varypataki ${ }^{\mathrm{b}}$, Elena A. Golovina ${ }^{\mathrm{c}}$, \\ Wim Jiskoot ${ }^{b}$, Geert-Jan Witkamp ${ }^{d}$, Young Hae Choi $^{a}$, and \\ Robert Verpoorte ${ }^{a}$ \\ ${ }^{a}$ Natural Products Laboratory, Institute of Biology, Leiden University, Leiden, The Netherlands \\ bivision of BioTherapeutics, Leiden Academic Centre for Drug Research, Leiden University, Leiden, \\ The Netherlands \\ ${ }^{c}$ Laboratory of Plant Physiology, Department of Plant Sciences, Wageningen University, Wageningen, \\ The Netherlands \\ ${ }^{\mathrm{d}}$ Division of Biological and Environmental Science and Engineering, King Abdullah University of Science \\ and Technology, Thuwal, Saudi Arabia \\ ${ }^{*}$ Corresponding author: e-mail address: dai_yuntao@live.cn
}

\section{Contents}

1. Introduction

2. Occurrence of NADES in plants 164

$\begin{array}{lll}2.1 & \text { Plants and stress } & 164\end{array}$

2.2 Flower petals containing pigments 164

$\begin{array}{ll}2.3 \text { Plants secretions } & 166\end{array}$

$\begin{array}{ll}2.4 \text { Plant seeds } & 167\end{array}$

3. The physicochemical properties of NADES 168

$\begin{array}{ll}3.1 \text { Liquid characteristics NADES } & 168\end{array}$

$\begin{array}{ll}3.2 \text { The diffusion of NADES in water } & 169\end{array}$

$\begin{array}{ll}\text { 3.3 Water content in cells and plants } & 171\end{array}$

3.4 The effect of NADES on the size, zeta potential and stability of liposomes 173

3.5 The effect of NADES on the membrane dynamics of liposomes 175

4. Conclusion 180

Acknowledgments 182

$\begin{array}{ll}\text { References } & 182\end{array}$

This chapter is an update from the nonpublished Chapter 8 of the $\mathrm{PhD}$ thesis of Dr. Yuntao Dai. 


\section{Abstract}

The components of natural deep eutectic solvents (NADES) are abundant in plants. This led to our hypothesis that NADES may play an important role in solubilizing, storing, and transporting poorly water-soluble metabolites in living cells, adjusting the water content of plants, and protecting cells when in harsh conditions. In order to test these hypothetical roles, diverse plant materials were analyzed, including leaves, petals, plant secretions and seeds. Comparatively high amounts of ingredients of NADES are observed in those organs. In particular, resurrection plants in dry state contain a higher amount of NADES components than fresh ones, and the level of NADES components is specifically higher in the outside layer (aleurone and seed cover) of barley, than in the inside (endosperm and embryo) layer. A high accumulation of sugars, sugar alcohols, amines, amino acids, and organic acids dominate plant secretions such as sap and nectar, often in typical molar ratios of NADES. This strongly supports the hypothesis of the existence of NADES in plants. For the roles, experimentally, NADES and water were mixed resulting in liquids with different compositions and properties. In the case of plants, NADES and water co-exist in the cells and may form ideal solvents for metabolites of diverse polarities and macromolecules. Some NADES are hygroscopic, providing evidence for possible water level controlling effects of NADES in plants. Most importantly, NADES may accumulate around the lipid bilayers, form intermolecular bonds with the polar heads of lipids, and stabilize the membrane, as revealed in experiments with liposomes. This study gives in vitro evidence for the different roles NADES may play in living organisms, and opens perspectives for further exploring the existence and functions of NADES in plants cells. The omics allows now to identify all molecules in an organism or even in a cell. The challenge for future research will be to understand how there molecules interact in the dynamic cellular processes and their compartmentation on a nanoscale. In other words the challenge is to unravel the molecular interactions in the three dimensions of space and the one of time, which will require a true multidisciplinary collaboration.

\section{Introduction}

Based on the observations made in metabolomics that all kind of microbial, plant and animal cells have similar patterns of some abundant primary metabolites in certain molar ratios, Choi et al. proposed the occurrence of a third liquid phase in living organisms. This liquid consists of two or more common solid (crystalline) primary metabolites that form an ionic liquid, or a deep eutectic solvent (Choi et al., 2011; Dai, van Spronsen, Witkamp, Verpoorte, \& Choi, 2013; Dai, Witkamp, Verpoorte, \& Choi, 2015). They called these liquids natural deep eutectic solvents (NADES). In fact, one may consider NADES as liquid supermolecules, composed of natural compounds in certain molar ratios connected by intermolecular interactions with each other, particularly H-bonding, i.e., NADES consist of a hydrogen 
bond donor and acceptor (Choi et al., 2011). The strong hydrogen bonding between these compounds is the crux for the specific characteristics of the NADES such as non-volatile, viscous and liquid in a wide temperature range. Their polarity is in the range of water and alcohols, making them particularly optimal solvents for medium polar compounds, which often have poor solubility in water, like most secondary metabolites.

When adding water to a NADES it is clear from the NMR spectra that until a dilution with about $50 \%$ of water, there is still an interaction between the NADES components. At higher dilutions the NMR spectra are like an aqueous solution of the NADES ingredients (Dai et al., 2015). In our view NADES are thus more a concept of the dynamics of different liquid phases in cells and organisms than a strict static physicochemical phenomenon of a deep eutectic mixture of two compounds. The components of NADES are metabolites that are found in high amounts in living cells (sugars, sugar alcohols, organic acids, amino acids, amines) as well as water. NADES possess some properties that make them very interesting solvents (Dai, van Spronsen, et al., 2013; Dai, Witkamp, Verpoorte, \& Choi, 2013, 2015), e.g., negligible volatility, low melting point (liquid even far below $-20^{\circ} \mathrm{C}$ ), broad polarity range and high solubilization power of a wide range of compounds (Choi et al., 2011; Dai, van Spronsen, et al., 2013, 2015). Poorly water-soluble low molecular weight metabolites and macromolecules (e.g., DNA, proteins, and polysaccharides) have shown good solubility in NADES (Mamajanov, Engelhart, Bean, \& Hud, 2010; Dai, van Spronsen, et al., 2013; Dai, 2013; Dai, Witkamp, et al., 2013; El Achkar, Fourmentin, \& Greige-Gerges, 2019; Núñez-Pertíñez \& Wilks, 2020, see chapter "Proteins in deep eutectic solvents: Structure, dynamics and interactions with the solvent" by Sanchez-Fernandez and Jackson). Moreover NADES are suitable for enzymatic reactions (Zhao, Baker, \& Holmes, 2011; Choi et al., 2011; chapter "Natural deep eutectic solvents as performance additives for biocatalysis" by Erol and Hollmann of this issue) and biotransformations (Gutiérrez, Ferrer, Yuste, Rojo, \& del Monte, 2010).

The hypothesis of NADES being a third liquid phase in organisms still needs more solid evidence. The indirect evidence comes from plant tolerance against, e.g., drought, cold, and salinity, in which tolerance has been found to be correlated with high levels of some of the mentioned common cell metabolites such as sugars (e.g., sucrose, trehalose, raffinose), sugar alcohols (e.g., inositol, mannitol), organic acids (e.g., ascorbic acid), amino acids (e.g., proline), and amines (e.g., betaine, choline) (Bartels \& Sunkar, 2005, chapters "NADES formation in vegetative desiccation tolerance: Prospects 
and challenges" by du Toit et al. and "Natural deep eutectic solvents-A new era of cryopreservation" by Jesus et al. of this issue). For example, the content of proline increased more than 10 times to nearly the same molar concentrations as sucrose during the natural desiccation of the resurrection plant Selaginella bryopteris (L.) Baker (Pandey et al., 2010). Whereas in other resurrection plants accumulation of high levels of glucose, sucrose and amino acids was observed, e.g., Sporobolus stapfianus Gand. (Whittaker, Martinelli, Farrant, Bochicchio, \& Vazzana, 2007), and of sucrose and raffinose in Xerophyta viscosa Baker (Peters, Mundree, Thomson, Farrant, \& Keller, 2007). Moreover, higher amounts of sugars (fructose, glucose, raffinose), proline, and galactinol were observed in Arabidopsis during cold acclimatization (Kaplan et al., 2007). In fact, in cryopreservation of tissues the common cryoprotectants are sugars, sugar alcohols, and proline (Mustafa, de Winter, van Iren, \& Verpoorte, 2011, see chapter "Natural deep eutectic solvents-A new era of cryopreservation" by Jesus et al. of this issue). Proline is important for the cold acclimatization of plants as well as for other organisms (Kovács et al., 2011). All these metabolites that correlate with drought, cold, or salinity have been classified as compatible solutes for organisms, i.e., non-toxic molecules which do not interfere with normal metabolism and accumulate predominantly in the cytoplasm at high concentration under various stress conditions (Bartels \& Sunkar, 2005; Yancey, Clark, Hand, Bowlus, \& Somero, 1982).

In our view, these compatible substances might be part of NADES that could be formed in various cellular compartments. Drought and freezing may result in the formation of a NADES phase containing proteins and low molecular weight compounds, whereas the water evaporates or freezes, respectively. This process keeps all the macro- and micro-molecules in different cellular compartments stored in a non-aqueous liquid phase, stabilized by a compartment specific NADES. Dilution with water, i.e., melting in case of frozen material or uptake in case of resurrection plants, restores the cellular structures and cells resulting in a living cell or organism.

Thus, the indirect or chemical evidence for the roles of NADES in living systems exists in the form of their high abundance in organisms, often in certain molar ratios like 1:1 or 2:1. However, real in situ occurrence of NADES in any type of organism still needs solid experimental proof. To prove their in situ presence will, first of all, require microscopy approaches. Assuming the presence of NADES in cells, however, one may predict certain physical characteristics of NADES that enables the interaction with other cellular components such as membranes, cell walls, and macromolecules including 


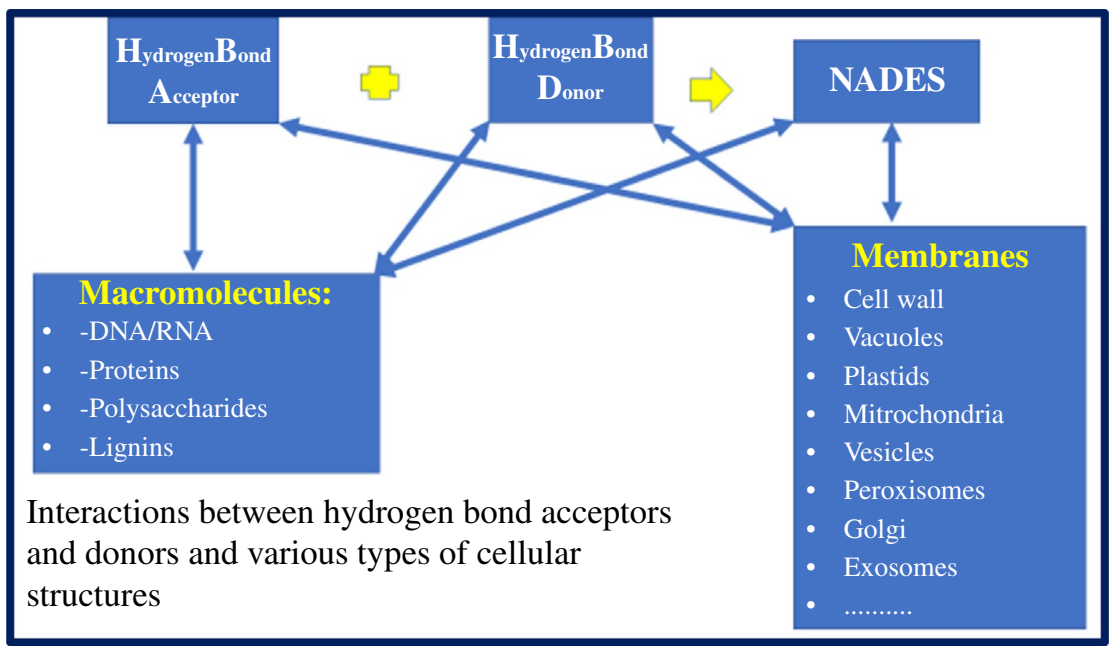

Fig. 1 Potential interactions between the hydrogen bond acceptor and hydrogen bond donor and various cellular structures.

polysaccharides, proteins, DNA, RNA and lignin. Particularly, the strong hydrogen bonding capacities of NADES components are an important characteristic in this context. A scheme of possible interactions of proton donor and proton acceptor molecules is shown in Fig. 1. Possibly all these interactions may occur in cells on a nanoscale, in a dynamic system. Some of these processes may play a role in an organism to overcome various forms of stress, like saline conditions, freezing temperatures, drought, wounding, and germination. Some NADES are part of static systems, like in seeds for long-term survival as well as in plants at low temperatures and in resurrection plants.

The aim of this chapter is to give an overview of the work we performed for the characterization of the biological roles of NADES found in plants. That includes both some previously reported and unpublished experimental data from the PhD thesis of Dr. Dai. Different plant materials and secretions have been collected and analyzed to obtain more evidence for the natural occurrence of NADES (Choi et al., 2011, see also chapters "Natural deep eutectic solvents present in plant exudates? A case study on the saps of Drosera species" by Vanda et al. and "Honey in traditional Chinese medicine: A guide to future applications of NADES to medicines" by Dai et al. of this issue). An interesting phenomenon of NADES is their potential to bind water, i.e., hygroscopicity could play a role in connection with drought-, and cold-stress as well as storage of seeds. As mentioned above, even up 
to a dilution with about $50 \%$ of water the components of NADES still interact with each other. This raises the question if a phase separation of NADES and water could occur, e.g., a layer of NADES adsorbed on membranes, proteins, polysaccharides, DNA or RNA being in equilibrium with an aqueous phase containing a host of different small molecules. Therefore, the diffusion process in a unstable two-phase water-NADES system was investigated to get information about the dynamics of such a system. Finally, to learn more about the interaction between NADES and membranes, the behavior of liposome bilayers in the presence of NADES in the media was studied.

\section{Occurrence of NADES in plants \\ 2.1 Plants and stress}

To study a possible role of NADES in resurrection plants, Selaginella pallescens (C. Presl) Spring was analyzed by ${ }^{1} \mathrm{H}$ NMR spectroscopy. These experiments showed that a comparatively high amount of the NADES components sugar (glucose), organic acids (succinic acid, tartaric acid, acetic acid, $\gamma$-aminobutyric acid), and amino acids (alanine, threonine, arginine) are present in dry $S$. pallescens if compared with fresh plant material (Dai, van Spronsen, et al., 2013). The increased levels of these metabolites as a reaction to drought was also reported for other plants, such as Arabidopsis, which showed increased levels of sugar (sucrose), amino acids (proline, alanine, arginine), organic acids (succinic acid, fumaric acid, malic acid), and an amine (choline) in water depleted conditions if compared with normal growing conditions (Dai, van Spronsen, et al., 2013). So, a relatively high amount of components of NADES exists in plants under dry conditions. However, to find direct evidence of the existence of NADES in plants to survive various harsh conditions, in situ analysis of plant materials on the levels of tissues, cells and cellular compartments would be required instead of the indirect evidence from the presence of NADES components in crude extracts of plant material.

\subsection{Flower petals containing pigments}

In cells, NADES might help to dissolve and preserve the non-water-soluble or non-water-stable compounds (e.g., many hydrophobic colorants) due to the fact that NADES solubilize non-water-soluble phenolic compounds (Dai, Verpoorte, \& Choi, 2014, 2015; Dai, Rozema, Verpoorte, \& Choi, 2016). 
Extremely high amount of rutin in the flower buds of Sophora japonica L. were reported to contain up to $22.9 \%$ (DW) of rutin (Couch, Naghski, \& Krewson, 1952). Paniwnyk, Beaufoy, Lorimer, and Mason (2001) optimized the extraction method and found about $12 \%$ of rutin in the flower bud material they analyzed. Considering its very low water solubility of rutin, the high rutin level in flower buds is a mystery, but a similar case has been reported for anthocyanins. Considering their solubility in water, a higher than expected concentration of anthocyanins was found in vacuolar inclusions (Markham et al., 2000). This might be explained by the presence of a high level of NADES in anthocyanoplasts and anthocyanic vacuoles. The co-existing NADES could dissolve high amounts of anthocyanins, and flavonoids, or even these phenolics may be part of a NADES itself. It is noteworthy that small amounts of water in NADES may improve the solubility of phenolics (Dai et al., 2015).

The types of NADES are expected to differ depending on tissue, organ, and even cellular compartment, connected with different roles of the NADES. In a metabolomics analysis of plant materials, one will thus find the ingredients for different NADES, which in the tissues, cells and cellular compartments could be clearly compartmentalized. Metabolomics analysis of extracts of plant materials does not give any information about the localization of the NADES ingredients. Only in situ analysis of the metabolites might be able to show that components of a NADES are together in the same space. For example, analyzing the extracts flowers of Catharanthus roseus (L.) G. Don a number of potential NADES components were found (Pan et al., 2014). To get insight into the molecular organization of the small molecules in the flowers, flower petals were directly measured by highresolution magic angle spinning (HR-MAS) ${ }^{1} \mathrm{H}$ NMR spectroscopy, a fast NMR spectroscopy method that is non-destructive and can be used to analyze biological materials without the need of extraction. It was expected that NADES components would be visible, as well as the interactions between these components, and even the interaction between NADES and the phenolics. However, the ${ }^{1} \mathrm{H}$ NMR spectrum of petals is dominated by sugars and organic acids (Fig. 2) and no signals of the phenolics were observed. Also, rutin, a major compound in the dry flower buds of Sophora japonica (upto $22.9 \%$, DW), was hardly detected in HR-MAS ${ }^{1} \mathrm{H}$ NMR using optimized conditions for small molecules (Dai et al. unpublished results). Further experiments are needed to also visualize the phenolics, which might be present in a bound form in a sort of polymeric state that is not visible with the applied HR-MAS NMR conditions for small molecules. Pohjala and 


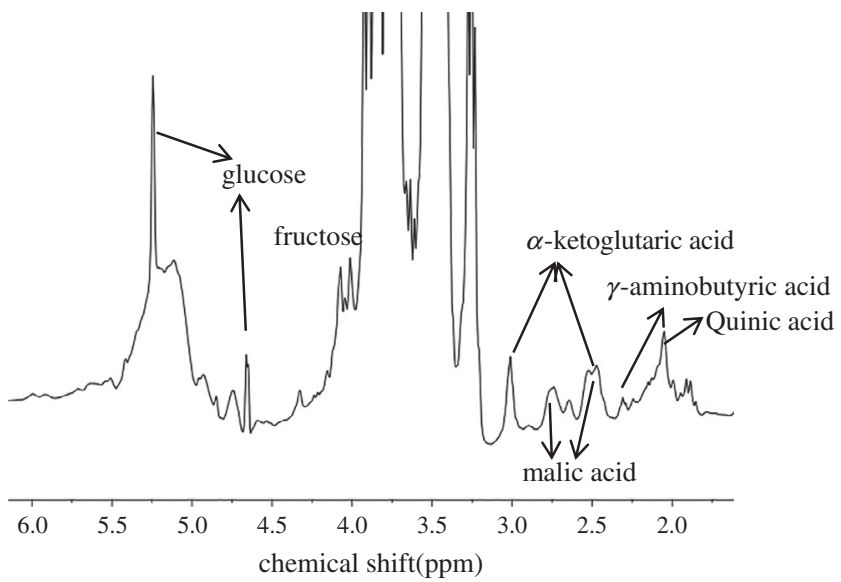

Fig. 2 High-resolution-magic angle spinning ${ }^{1} \mathrm{H}$ NMR spectrum $(400 \mathrm{MHz})$ of the red petals of Catharanthus roseus.

Tammela (2012) reported that flavonoids aggregate easily, which may affect, for example, various bioassays. Scheidt, Pampel, Nissler, Gebhardt, and Huster (2004) showed that flavonoids interact with artificial membranes in a freeze dried preparation of liposomes and flavonoids by using MAS ${ }^{1} \mathrm{H}$ NOESY NMR spectroscopy. These observations might be an interesting model for studying the effect of NADES on the interaction of the phenolics and membranes, e.g., in cryopreservation experiments and in resurrection plants.

It is difficult to detect NADES itself in plants. The dynamic changes of NADES in cells and the presence of different potential ingredients of NADES in tissues, cells or cellular compartments, such as vacuoles, vesicles, exosomes, peroxisomes, mitochondria, or plastids point to the possibility that different NADES may occur in different cellular compartments. That means on a nanolevel. This requires further study on the cellular localization of NADES and NADES ingredients, i.e., on nanolevel.

\subsection{Plants secretions}

Various secretions, such as maple syrup, nectar, and mucilages are present extracellularly in plants. For example, sucrose:fructose:glucose with a molar ratio around 1:1:1 was detected in the nectar of Cleome houtteana Schltdl. (synonym Cleome hassleriana Chodat), and honey is composed of glucose and fructose (1:1, molar ratio) (Choi et al., 2011). In chapter "Natural deep 
eutectic solvents present in plant exudates? A case study on the saps of Drosera species" by Vanda et al. of this issue some further studies on various exudates of plants are discussed.

\subsection{Plant seeds}

A seed, as a temporary static system, is an interesting model to study the presence of NADES. In our viewpoint, NADES could be involved in the cold and drought resistance of seeds and keeping seeds viable in dormant stage, i.e., germinate when under the right conditions. In this context, NADES could keep essential enzymes dissolved and upon addition of water these enzymes are activated (Choi et al., 2011). The ${ }^{1} \mathrm{H}$ NMR of extracts from the outside and inside parts of barley seeds revealed the presence of, among others, sucrose, organic acids (acetic acid, succinic acid), amino acids (threonine, alanine), alcohols (isobutanol, ethanol), and amines (choline, betaine) (Fig. 3) (Choi et al., 2011). Interestingly, the outside layer (aleurone and seed cover) showed about equal molar amounts of sucrose and choline as major components. These may be present as a NADES in which some of the germination related enzymes are dissolved. These enzymes may become active after dilution of the NADES (Choi et al., 2011). Schuurink, Sedee, and Wang (1992) emphasized the need for protection of enzymes stored in the outside layer of the seed that must survive in extremely dry conditions during the seeds dormant period. NADES have been shown to well dissolve

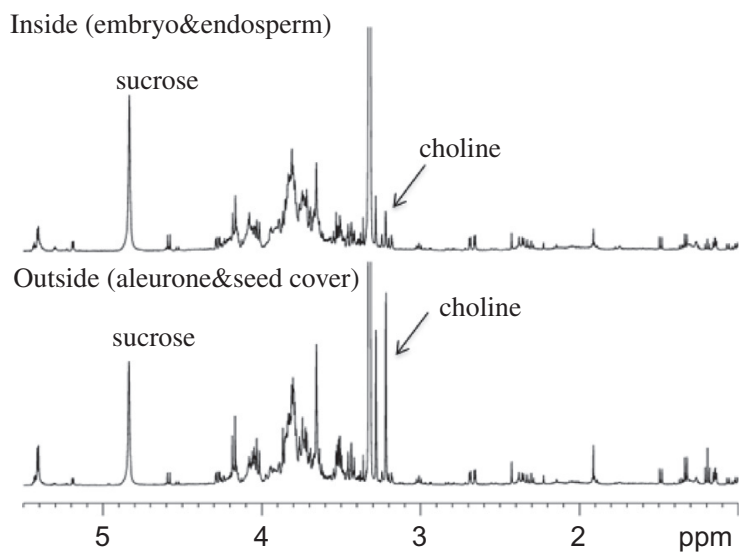

Fig. $3{ }^{1} \mathrm{H}$ NMR spectra $(500 \mathrm{MHz})$ of extracts made with $50 \% \mathrm{CH} 3 \mathrm{OH}-d_{4}$ in buffer $(90 \mathrm{mM}$ $\mathrm{KH}_{2} \mathrm{PO}_{4}$ in $\mathrm{D}_{2} \mathrm{O}$ ) containing $0.05 \%$ TMSP (trimethylsilyl propionic acid sodium salt $(\mathrm{w} / \mathrm{v})$ ) (A) the inside and (B) outside parts of barley seeds (aleurone). 
proteins and to stabilize them (Arakawa \& Timasheff, 1982; Choi et al., 2011, see also chapters "Proteins in deep eutectic solvents: Structure, dynamics and interactions with the solvent" by Sanchez-Fernandez and Jackson and "Natural Deep Eutectic solvents as performance additives for biocatalysis" by Erol and Hollmann in this issue).

\section{The physicochemical properties of NADES}

\subsection{Liquid characteristics NADES}

Several physicochemical properties of NADES were measured: the density of NADES lies in the range of $1.08-1.36 \mathrm{~g} / \mathrm{cm}^{3}$ (Dai, van Spronsen, et al., 2013) and their viscosity between 37 and $720 \mathrm{~mm}^{2} / \mathrm{s}$, which is much higher than water $\left(1 \mathrm{~mm}^{2} / \mathrm{s}\right)$. The viscosity substantially increases at low temperature or with low water contents. The NADES have a low $T_{\mathrm{g}}$ (temperature of glass formation) far below $-50{ }^{\circ} \mathrm{C}$, thus they are stable liquids over a wide temperature range.

Several studies have reported the physicochemical properties of cytoplasm in cells before the intracellular molecular glass formation in plant anhydrobiosis. The cytoplasmic glass in dry cells results from a complex of sugars and other cytoplasmic components such as organic acids, amino acids, and salts (Buitink \& Leprince, 2004). The viscosity of cytoplasm increases during drying-out conditions and eventually reaches a glass state. In general, a glass has the following physical properties: high density, temperature dependent mobility and slow mobility in solid state, high viscosity, and low $T_{\mathrm{g}}$ (Buitink \& Leprince, 2008; Dijksterhuis, Nijsse, Hoekstra, \& Golovina, 2007), which are also typical for the physical properties of NADES (Dai, van Spronsen, et al., 2013). The increasing levels of several NADES constituents in plants during anhydrobiosis attest a role of NADES in protecting the cells and tissues, e.g., in stabilizing the essential proteins and the various cellular membranes (see below). In fact, freezing of cells or plants would result in a similar process, in which water will freeze as pure water, leaving all compounds, both of small and high molecular weight, in the cellular liquids as a rest phase like in case of anhydrobiosis. Freezing resistance could thus be built upon the production of high levels of NADES ingredients. In fact, in cryopreservation of plant cells, typical cryoprotectants added to improve the chances of survival of the plant cells are all potential NADES components (Mustafa et al., 2011, see also chapter "Natural deep eutectic solvents-A new era of cryopreservation" by Jesus et al. in this issue). 


\subsection{The diffusion of NADES in water}

Most NADES are hydrophilic and are miscible with water since the individual components are polar compounds. The high viscosity of NADES slows down the diffusion of water in a NADES. An example of a high viscosity NADES is sucrose-choline chloride-water $(1: 4: 4)(\mathrm{SuCH})$, whereas 1,2propanediol-choline chloride-water $(1: 1: 1)(\mathrm{PpCH})$ is an example of a low viscosity NADES (Dai, van Spronsen, et al., 2013). To study the diffusion in a two-phase system consisting of NADES and water, experiments were performed with either of these NADES as the lower layer, on top of which water was carefully pipetted. In case of equal diffusion rates of the constituents in both layers, one would expect the disappearance of the interface when both layers have become identical. If the diffusion rates in both layers are not equal, the interface would move up (water diffuses faster into lower NADES layer, than NADES into the water layer) or down (NADES components have faster diffusion in upper layer, than water in the lower layer). To be able to follow the diffusion process over time, carthamin was added as dye in either phase. The diffusion between the NADES and water behaved differently than expected, as instead of two phases, three phases (upper, middle and lower) were observed (Fig. 4, day $10 \mathrm{SuCH}$;

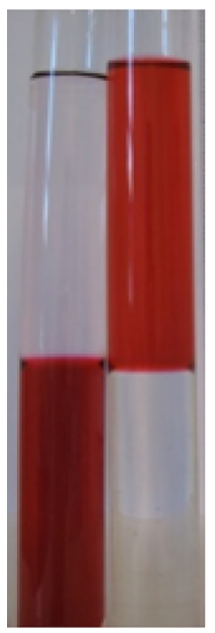

day 0

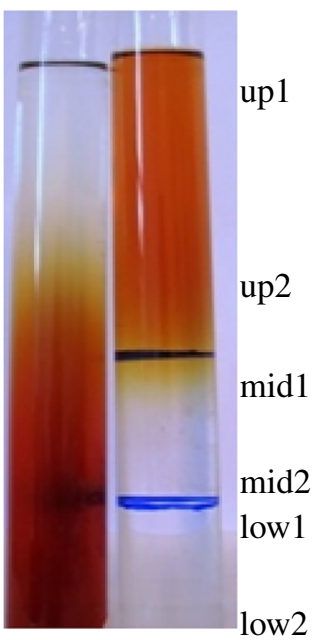

day 10

Fig. 4 A picture of diffusion tests between sucrose-choline chloride-water (1:4:4) and water on day 0 and 10, and 3 layers (upper, middle, and lower) were observed with two interfaces labeled in blue on day 10 (left: carthamin in the sucrose-choline chloride layer; right: carthamin in the water layer). 
the two interfaces marked with blue color to improve the visibility). Apparently, the dye diffuses from the lower layer to the middle and from there to the top layer, whereas little diffusion of the dye occurs from the top layer to the lower layers, though the solubility of the dye is higher in the NADES than in water (Dai, 2013; Dai et al., 2014).

The same results were observed with the low viscosity $\mathrm{PpCH}$, though the intermediate layer disappeared already in 2 days. So, the dye diffusion between $\mathrm{PpCH}$ and water was much faster than that from $\mathrm{SuCH}$ and water. Faster diffusion was also observed when the starting phase of $\mathrm{SuCH}$ was diluted with $10 \%(\mathrm{v} / \mathrm{v})$ water. Viscosity clearly plays a complex role in the phase behavior between NADES and water.

To learn more about the composition of the intermediate phase, samples were collected from the upper and the lower phase, as well as of two different levels of the middle layer. The water content of each sample was determined and the organic components were quantified by means of ${ }^{1} \mathrm{H}$ NMR (Table 1). At day 10, the water content in the water layer showed a gradient from about $90 \%$ to $60 \%$ (up to down), in the middle layer 50\%-20\% and more or less unchanged in the lower phase, showing that instead of complete mixing, a new metastable NADES was formed as an intermediate phase (Table 1). The ratio of the two components in NADES is different in the layers. The molar ratio of choline chloride to sucrose in $\mathrm{SuCH}$ is 4 when prepared. During the diffusion process, the ratio was around 15 in the upper layer, 3 in the middle layer, and remained at the same value of 4 in the lower

Table 1 The water weight percentage and molecular ratio of components of sucrosecholine chloride in two parts of three different layers (upper, middle, and lower) after 10 days' diffusion between water and sucrose-choline chloride-water (1:4:4) $(n=3)$ (see Fig. 4).

\begin{tabular}{|c|c|c|c|c|c|c|c|}
\hline & & up1 & up2 & mid1 & mid2 & low1 & low2 \\
\hline \multirow[t]{3}{*}{ Water w\% } & 1 & $90 \pm 2.2$ & $60.1 \pm 3.3$ & $50.0 \pm 4.5$ & $18.0 \pm 3.7$ & $9.0 \pm 2.8$ & $6.4 \pm 1.5$ \\
\hline & 2 & $91.9 \pm 1.7$ & $64.2 \pm 5.7$ & $42.1 \pm 4.5$ & $15.3 \pm 2.7$ & $6.4 \pm 1.0$ & $6.3 \pm 0.9$ \\
\hline & 3 & 90 & 62 & 49 & 15 & 7.9 & 7.2 \\
\hline \multirow{3}{*}{$\begin{array}{l}\text { Molar ratio } \\
\text { (choline: } \\
\text { sucrose) }\end{array}$} & 1 & 14.5 & 3.2 & 2.8 & 3.1 & 3.5 & 3.7 \\
\hline & 2 & 16.6 & 3.0 & 3.0 & 3.5 & 3.5 & 3.9 \\
\hline & 3 & 10.3 & 3.0 & 2.7 & 3.1 & 3.5 & 3.7 \\
\hline
\end{tabular}

NADES components were quantified by means of ${ }^{1} \mathrm{HNMR}$ measurements. 
layer. Apparently, in the interface of $\mathrm{SuCH}$ and water a new phase is formed from which choline can move into the water layer whereas the sugar is kept in the middle layer by the hydrogen bonding.

This mixing behavior indicates the complexity of the physicochemical properties of the cellular contents. A change in $\mathrm{pH}$, concentration, temperature, etc., may cause the formation of separate phases. In a dynamic system like the endoplasmic reticulum, conditions for the biosynthesis of poorly water-soluble compounds may be created transiently via the formation of a third NADES phase in which enzymes are dissolved and where poorly water-soluble substrates are concentrated from the cytosol. Metabolons could thus be formed in this way (see chapter "Metabolons and biocondensates: The essence of plant plasticity and the key elements in development of green production systems" by Møller and Laursen). Layers of NADES might even be formed on membranes in a dynamic equilibrium with the liquid phase surrounding the membrane.

\subsection{Water content in cells and plants}

Some NADES we noted are hygroscopic. That raised the question if NADES in plants may play a role in controlling water levels, e.g., by absorbing water from the surroundings. Hygroscopicity measurements of glucose-choline chloride-water (2:5:5) (GCH) showed gradual increases of water content with an increasing humidity level in the surroundings from $0 \%$ to $80 \%$. The process is reversible when the humidity goes down (Fig. 5A). The water percentage in $\mathrm{GCH}$ reached equilibrium in about $10 \mathrm{~h}$ for a certain condition (Fig. 5B). So, the NADES may function in adjusting the water level in a plant through interaction with water vapor in the air.

To explore a hygroscopicity effect in plants, the water level of a barley seed was tested at a relative humidity level of $80 \%$ at $25^{\circ} \mathrm{C}$ (Fig. 6). As can be seen, most water was absorbed during the first $48 \mathrm{~h}$ and the equilibrium water content for $80 \%$ external relative humidity was about $9.1 \%(\mathrm{w} / \mathrm{w})$. When the humidity level decreased to zero, the amount of absorbed water in the barley seed returned to the initial level within the same time interval. The observation fits our hypothesis of NADES being involved in water loss and uptake, e.g., several NADES are clearly hygroscopic and at the same time are able to strongly retain certain minimum amounts of water. The barley aleurone has been shown to contain sucrose and choline in an about 


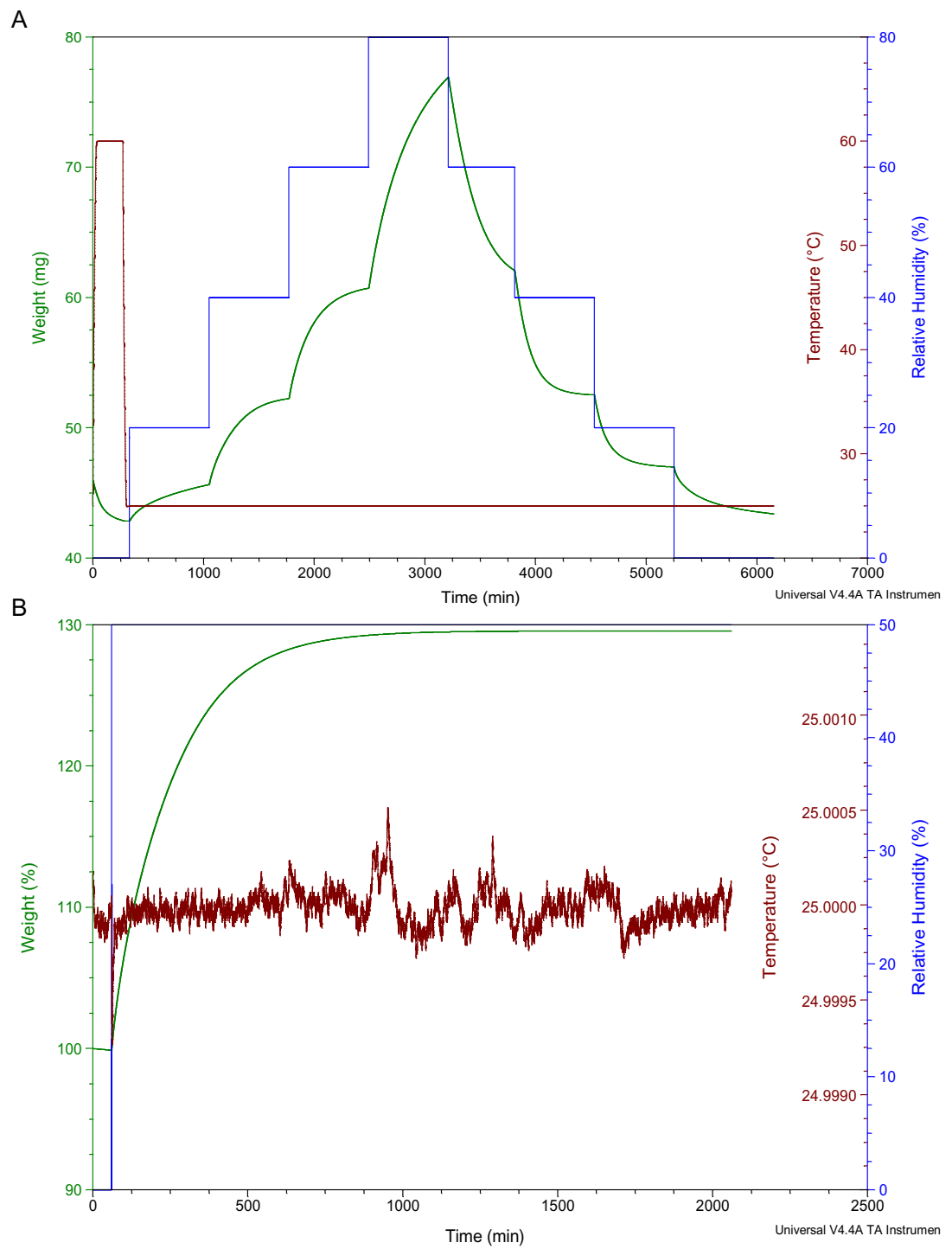

Fig. 5 Hygroscopicity experiment of glucose:choline chloride:water $(1: 2.5: 2.5)$ at $25^{\circ} \mathrm{C}$ with relative humidity steps (A) and $50 \%$ relative humidity level (B). The green line indicates the weight of glucose:choline chloride:water and the blue line refers to the relative humidity of test conditions.

1:1 M ratio. The NADES sucrose-choline chloride was found to be hygroscopic (Dai et al., unpublished results). The real in situ molecular organization of the aleurone is not known, so we cannot conclude yet if indeed these components are present as a NADES. But, apparently, plants can lose water 


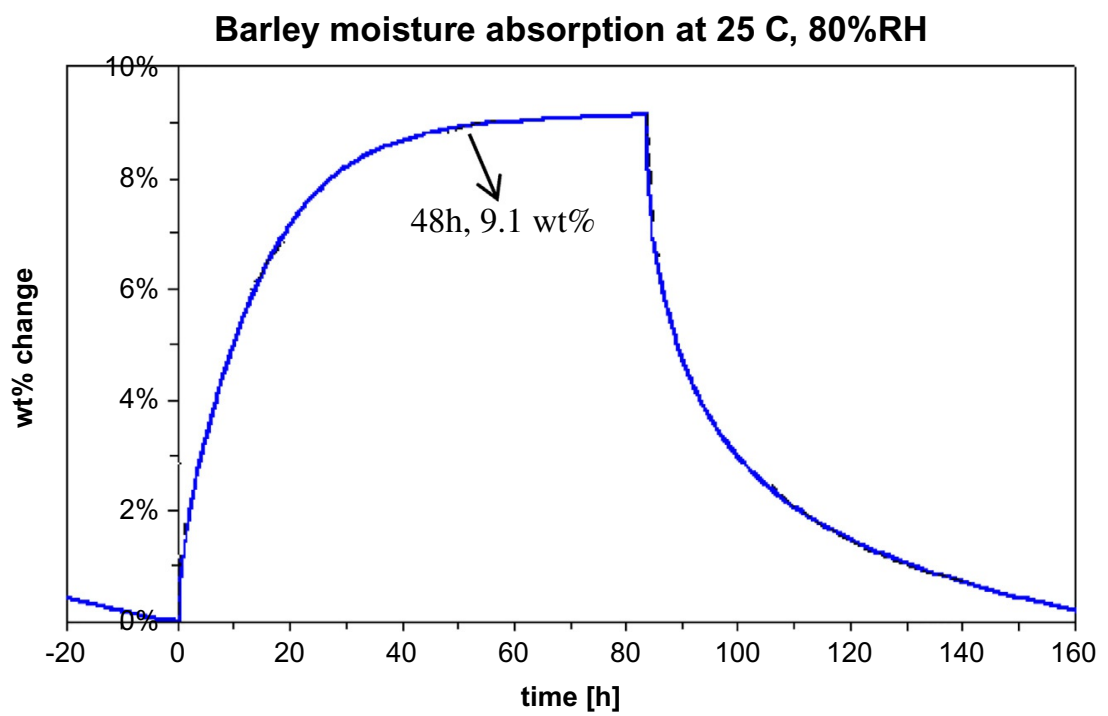

Fig. 6 The hygroscopicity experiment with intact barley seeds at $25^{\circ} \mathrm{C}$ with $20 \mathrm{~h}$ drying, followed by $84 \mathrm{~h} 80 \%$ relative humidity level, and then back to $0 \%$ relative humidity level.

in relative dry conditions and absorb water in relative humid conditions. The hygroscopicity may explain how mosses can live on rocks, and how cacti can survive in deserts. Cacti open their stomata during the night which allows the exchange of oxygen and carbon dioxide. But at the same time with increasing relative humidity of the air due to low night temperatures, cacti may also catch the water molecules via a hygroscopic NADES. That NADES could be formed during the day time from sugars produced from the carbon dioxide and water that were absorbed during the night. The water uptake may activate the enzymes of photosynthesis that subsequently produce sugars again during the day thus restoring a hygroscopic NADES for nightly water harvesting.

\subsection{The effect of NADES on the size, zeta potential and stability of liposomes}

As mentioned above, our hypothesis is that NADES could be attached to cellular membranes by hydrogen bonding and hydrogen accepting interactions with the polar head groups in the lipid membranes. Therefore, the effect of some NADES on liposomes was tested as a model for the interaction of various cellular membranes with NADES. In these experiments artificial liposomes were made with $7.8 \mathrm{mmol}$ 1,2-dioleoyl-3-trimethylammoniumpropane (chloride salt), $3.9 \mathrm{mmol}$ soybean $\mathrm{L}-\alpha$-phosphatidylcholine, and 
Table 2 The size (hydrodynamic diameter, $\mathrm{nm}$ ) and zeta potential (ZP) of liposome in three media $(n=3)$ : phosphate buffer $(\mathrm{pH}=7.0)$, buffer with $18.0 \%(\mathrm{w} / \mathrm{w})$ sucrose:malic acid (SM), buffer with $27.8 \%(\mathrm{w} / \mathrm{w}$ ) sucrose:fructose:glucose (SFG) and buffer with $18.2 \%$ $(\mathrm{w} / \mathrm{w})$ sucrose-proline $(\mathrm{SPr})$

\begin{tabular}{lllllll} 
& \multicolumn{3}{c}{ Size $(\mathbf{d}, \mathbf{n m})$} & & \multicolumn{2}{c}{ ZP } \\
\cline { 2 - 4 } \cline { 7 - 7 } & Average & PDI $^{\mathrm{a}}$ & RSD $\%$ & & Average & RSD\% \\
\hline Buffer + SM & 117.2 & 0.124 & 0.60 & 40.5 & 0.65 \\
\hline buffer + SFG & 192.2 & 0.175 & 4.85 & 20.4 & 12.70 \\
\hline Buffer + SPr & 790 & 0.369 & 25.90 & 15.2 & 34.30 \\
\hline Buffer & 162.5 & 0.092 & 1.07 & 27.4 & 1.03 \\
\hline
\end{tabular}

${ }^{a}$ PDI: polydispersity index, the width of molecular size distribution.

$3.9 \mathrm{mmol}$ cholesterol. The liposomes were kept in a phosphate buffer of $\mathrm{pH}$ 7. When NADES was added, the size (hydrodynamic diameter) of liposomes was increased by $30 \mathrm{~nm}$ in a buffer with $27.8 \%$ sucrose:fructose:glucose $(1: 1: 1)$ (SFG), increased by $600 \mathrm{~nm}$ in buffer with $18.2 \%$ sucrose:proline (1:1) SPr, and decreased by $40 \mathrm{~nm}$ in buffer with $18.0 \%$ sucrose:malic acid (1:1) (SM), compared with phosphate buffer (Table 2). The levels of the NADES are such that the liquid behaves like an aqueous solution, i.e., have a similar viscosity as the buffer (Dai, van Spronsen, et al., 2013). It means that NADES do affect liposomes, they may attach to them, or vacuoles may aggregate which increases the size of liposomes in the case of SFG and SPr, whereas SM decreases the liposome size.

The zeta potential decreased in buffer with SFG, SPr and increased in buffer with SM, compared with phosphate buffer. The different zeta potentials confirm interactions between NADES and liposomes and also imply that the interactions between liposomes and various NADES are different. For SFG, the sugar components may hydrogen bond to the head group of the lipids and form a film around the liposome through a hydrogen bonding network, increasing the size of liposome (Crowe, Hoekstra, \& Crowe, 1992). Moreover the covering of the liposome with a neutral NADES may explain the lower zeta potential. For SM, the negatively charged acid group of malic acid may have ionic interactions with the positively charged head group of the lipids and this interaction may shrink the size of liposome due to the attraction effect. The size of liposomes with SPr increased but they were physically unstable probably due to the zwitterionic form of proline. The preparation of liposomes with sucrose: choline chloride (SC) in 
buffer ( $\mathrm{pH}$ 7.0) was also attempted but the hydration of the lipid film failed and the lipid precipitated in the buffer containing SuC. This might be because of the repulsive forces between choline and the head group of the lipid (1,2-dioleoyl-3-trimethylammonium-propane). In all, the different behavior of liposomes in buffers containing NADES with neutral (SFG), acid (SM), basic (SC) and zwitterionic (SPr) components indicates the existence of interactions between liposomes and the molecules of NADES components.

The stability of liposomes in different media was investigated with the size and zeta potential as parameters. The size, polydispersity index, and zeta potential of liposomes were practically stable for 1 month at $4{ }^{\circ} \mathrm{C}$ in buffer and buffer with SFG or SM, but unstable in SPr. This indicates that liposomes are stable for shape, homogeneity, and interaction between NADES and liposomes for at least 1 month in buffer, buffer with SFG or SM. However, after 5 months at $4{ }^{\circ} \mathrm{C}$, the liposomes had precipitated in both buffer and SPr, while they were still in suspension in SFG and SM. This proves a stabilizing effect of some NADES on liposomes. The stabilizing effects of sugars on membranes and liposomes have been known already for many years (Crowe et al., 1988, 1992; Crowe, Crowe, Carpenter, \& Aurell Wistrom, 1987; Crowe, Leslie, \& Crowe, 1994). Further studies should establish if the NADES effects are different from the sugar effect. The strong proton donor/proton acceptor interactions in the NADES may lead to the formation of a NADES layer on membranes, in which even selectivity may play a role, e.g., a different binding with an acid (e.g., malic acid) or a base (e.g., choline) containing NADES, depending on the character of the membranes.

\subsection{The effect of NADES on the membrane dynamics of liposomes}

The effect of NADES on membrane dynamics was studied with the EPR spin probe technique. A spin label 5-doxylstearic acid (5-DS) was used to probe the membrane interface in liposomes. In the 5-DS molecule the nitroxide doxyl group (a stable radical) is attached in a rigid, stereospecific manner to stearic acid at the fifth carbon from the $\mathrm{COOH}$ group. In phospholipid membranes the $\mathrm{COOH}$ group of spin-labeled stearic acid is inserted between the polar heads. Therefore, the nitroxide (doxyl group) of 5-DS resides in a polar area of the bilayer, which is called the bilayer surface or bilayer interface. The EPR spectral shape of 5-DS depends on the motion and angular orientation of the nitroxide group with respect to the 
membrane lipid-water interface (Marsh, 1981). This spin label allows the probing of the motional freedom in membranes at the lipid-water interface of the bilayer.

The anisotropic character of the spectral shape of 5-DS in liposomes in normal buffer (Fig. 7A) results from the restricted angular freedom of the radical group of 5-DS in the bilayer interface. The spectral parameters $A_{\max }$ and $A_{\min }$ indicate the outer and inner hyperfine splitting in an experimental spectrum as shown in Fig. 7A. The membrane-order parameter $S_{z z}$ can be calculated as the ratio between the observed hyperfine anisotropy $\left(A_{\max }-A_{\min }\right)$ to the maximum theoretically obtainable value, which corresponds to the completely rigid orientation of 5-DS (Knowles, Marsh, \& Rattle, 1976). With membrane fluidization outer splitting $\left(\mathrm{A}_{\max }\right)$ decreases and inner splitting $\left(A_{\min }\right)$ increases, so that the order parameter decreases. Later on, some corrections have been proposed to account for differences in polarity and for the range of membrane-order parameter. Here the order parameter is calculated according to the formula proposed by Marsh and Schorn (1998). This formula takes into account the principle splitting values for 5-DS and is corrected for polarity of the spin label environment. $\mathrm{S}_{\mathrm{zz}}=1 / 7\left(\mathrm{~A}_{\max }+2 \mathrm{~A}_{\min }\right)-\operatorname{sqrt}\left\{\left[1 / 7\left(\mathrm{~A}_{\max }+2 \mathrm{~A}_{\min }\right)\right]^{2}-0.46\left(\mathrm{~A}_{\max }-\mathrm{A}_{\min }\right)\right.$ $+0.6\}$.

The EPR spectra of the 5-DS incorporated into the liposome in four media were compared: phosphate buffer, phosphate buffer with SFG (27.8\%), phosphate buffer with SM (18.0\%), and phosphate buffer with $\mathrm{SPr}(18.1 \%)$. The data on $2 \mathrm{~A}_{\max }$ and $\mathrm{S}_{\mathrm{zz}}$ are presented in Table 3. Outer splitting $2 \mathrm{~A}_{\max }$ considerably decreases in the presence of $18.0 \% \mathrm{SM}$ and decreases insignificantly in the presence of SFG and SPr. The order parameter $\mathrm{S}_{\mathrm{zz}}$ of the EPR spectra of 5-DS in liposomes in the presence of $18.0 \%$ $\mathrm{SM}$ is considerably reduced (Table 3). In the presence of SFG and SPr the order parameter slightly increases.

The decrease of outer splitting $2 \mathrm{~A}_{\max }$ and order parameter $\mathrm{S}_{\mathrm{zz}}$ in the presence of SM result from the fluidization of the membrane interface. This data is also supported by a decrease in the width of the central line (Table 3). The results are in accordance with a considerable decrease of liposome size from 162.5 to $117.2 \mathrm{~nm}$ (Table 2). Obviously, the decrease of liposome size inevitably causes the fluidization of the liposome bilayer outer surface due to an increased bilayer curvature and the spacing between lipids. The effect of bilayer curvature on phospholipid behavior has been demonstrated by molecular dynamics simulations (Risselada \& Marrink, 2009). The size of liposomes in the presence of SFG and SPr considerably increases (Table 3). However, 


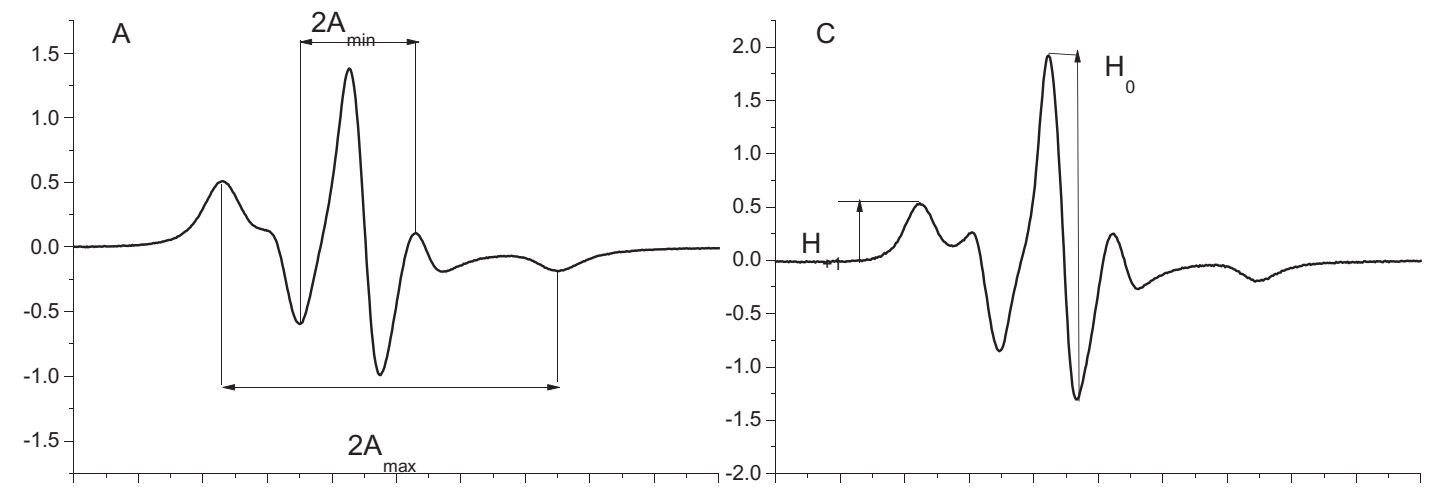

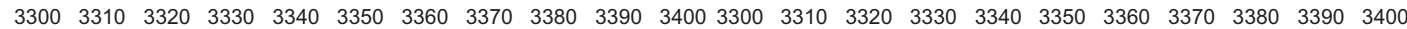
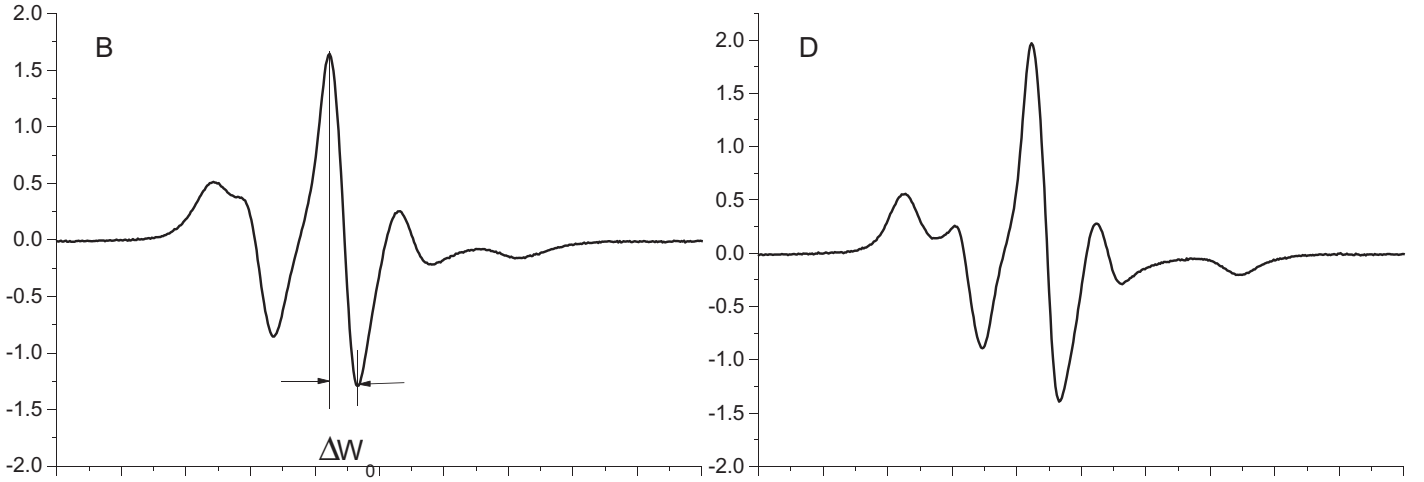

$\begin{array}{llllllllllllllllllllll}3300 & 3310 & 3320 & 3330 & 3340 & 3350 & 3360 & 3370 & 3380 & 3390 & 3400 & 3300 & 3310 & 3320 & 3330 & 3340 & 3350 & 3360 & 3370 & 3380 & 3390 & 3400\end{array}$

Field, gauss

Fig. 7 Electron paramagnetic resonance spectra of 5-doxyl stearic acid in liposomes in PBS phosphate buffer (pH 7.0) (A); in PBS phosphate buffer with $18.0 \%(\mathrm{w} / \mathrm{w})$ sucrose:malic acid: water (SM) (B); in PBS phosphate buffer with $27.8 \%(\mathrm{w} / \mathrm{w})$ sucrose:fructose:glucose (SFG) (C); and in PBS phosphate buffer with $18.1 \%(\mathrm{w} / \mathrm{w})$ sucrose:proline (SPr) (D). 
Table 3 Parameters of electron paramagnetic resonance spectra of 5-doxyl stearic acid in liposomes in the presence of three different natural different deep eutectic solvents (buffer with $18.0(\mathrm{w} / \mathrm{w})$ sucrose:malic acid $(\mathrm{SM})$, buffer with $27.8 \%(\mathrm{w} / \mathrm{w})$ sucrose: fructose:glucose (SFG), and buffer with $18.1 \%(\mathrm{w} / \mathrm{w})$ sucrose:proline (SPr), compared with phosphate buffer $(\mathrm{pH}=7.0))$.

\begin{tabular}{|c|c|c|c|c|}
\hline Media composition & $2 A_{\max }$ (Gauss) & $\mathrm{S}_{\mathrm{zz}}$ (Rel. units) & $\Delta \mathrm{W}_{0}$ (Gauss) & $\begin{array}{l}\mathrm{H}_{0} / \mathrm{H}_{+1} \\
\text { (Rel. units) }\end{array}$ \\
\hline Buffer $(\mathrm{pH}=7.0)$ & 52.08 & 0.617 & 4.6919 & 2.71391 \\
\hline Buffer + $27.8(\mathrm{w} / \mathrm{w})$ SFG & 51.99 & 0.629 & 4.30102 & 3.67734 \\
\hline Buffer $+18.0 \%(\mathrm{w} / \mathrm{w}) \mathrm{SM}$ & 47.45 & 0.507 & 4.30103 & 3.26143 \\
\hline Buffer $+18.1 \%(w / w) S P r$ & 51.87 & 0.622 & 4.30102 & 3.55936 \\
\hline
\end{tabular}

The parameters include membrane-order parameter $\left(\mathrm{S}_{\mathrm{zz}}\right)$, the outer hyperfine splitting $\left(2 \mathrm{~A}_{\max }\right.$, in gauss), the width of the central line $\left(\Delta \mathrm{W}_{0}\right.$, in gauss) and the ratio between the heights of the central $\left(\mathrm{H}_{0}\right)$ and low-field $\left(\mathrm{H}_{+1}\right)$ lines $\left(\mathrm{H}_{0} / \mathrm{H}_{+1}\right.$, relative units).

this causes only a slight ordering of the membrane interface as determined by order parameter (Table 3). If the ordering/immobilization increases slightly in the presence of SFG and SPr, an increase of the outer splitting $2 \mathrm{~A}_{\max }$ would also be expected. However, this was not the case. The slight decrease of the $2 \mathrm{~A}_{\max }$ for these cases could be explained by the decrease of the polarity in the vicinity of spin label moiety.

The width of the central line decreases in the presence of all NADES (Table 3, Fig. 7), while the ratio between the heights of the central $\left(\mathrm{H}_{0}\right)$ and low-field $\left(\mathrm{H}_{+1}\right)$ lines (as indicated in Fig. 6C) increases in the presence of all NADES (Table 3, Fig. 8). There are many factors that determine the line width in the EPR spectra. Line broadening can be paramagnetic and motional. Line narrowing can only be explained in the case of SM by fluidization of the membrane interface, because in this case the order parameter of the spectrum also decreases. In the case of SFG and SPr the situation is more complicated. Both spectra are identical, thus the mechanism of line narrowing for these two NADES must be the same. In these samples, line narrowing occurs without the decrease of the order parameter. The order parameter relates to the average angle of the departure of the molecular axis of the acyl chain from the normal to the bilayer surface (angular orientation or angular freedom). When the system is disordered, this angle increases and the spin label moiety experiences more freedom to move. This results in line narrowing. If order parameter does not change, this can only mean that the rate of rotation around the molecular axis of the acyl chain increases without system disordering. Usually such effect is observed in the membrane 


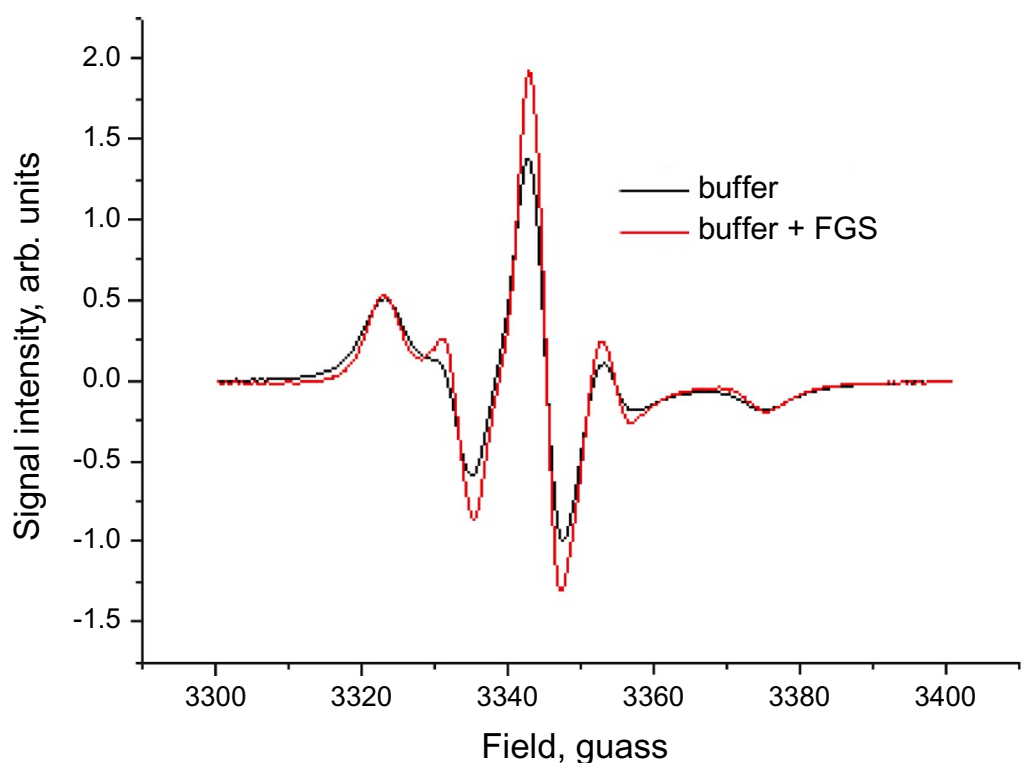

Fig. 8 Comparison of the electron paramagnetic resonance spectral shape of 5-doxyl stearic acid in liposomes in buffer (black lines) and in buffer with SFG (red lines).

interface at higher temperature. The fact that the same phenomenon is observed in case of NADES at room temperature, is very intriguing.

If line narrowing originates from a decreased probability of interaction between the spin label moieties at the liposomal membrane (paramagnetic broadening due to dipole-dipole interaction or exchange), then such an effect may result from decreased membrane lateral diffusion (Sachse, King, \& Marsh, 1987). The restriction of lateral diffusion of lipids within bilayers in the presence of NADES would be one other possible mechanism for their stabilizing effect. All the parameters in EPR show an unexpected behavior of 5-DS which may indicate the establishment of a NADES layer around the liposomes. Generally spin label experiments are in aqueous surroundings, with the $-\mathrm{COOH}$ group of $5-\mathrm{DS}$ at the polar heads of the lipid bilayer and the doxyl group in a polar area of the bilayer. If NADES builds up a shell around the liposomes, the label will be in NADES, a less polar but viscous environment, in which the 5-DS might bind more loosely with the polar heads of the bilayer as it dissolves better in the NADES than in water. In other words, the 5-DS will have a different behavior in a water-lipid system than in a water-NADES-lipid system due to a different interface at the bilayer. The decrease of the line width and increase of the $\mathrm{H}_{0} / \mathrm{H}_{+1}$ ratio in 
the spectra of 5-DS in liposomes in the presence of SFG and SPr are a clear indication of the presence of another fraction of 5-DS, which demonstrates liquid-type behavior. These spectra may be considered as the sum of two spectra-one originates from the immobilized doxyl group (anisotropic spectrum), another-from the freely rotating spin label (isotropic spectrum with narrow lines). Superposition of two spectra results in the central line narrowing together with almost non-changed order parameter. The interaction of 5-DS with the bilayer is not homogenous, the differences may be caused by different localizations of the spin-label moiety in the bilayer. One localization might allow the free rotation of the doxyl group, the other location might be one more embedded in the bilayer. The isotropic spectrum might also result from any local spacing in the bilayer due to insertions of some molecular groups between phospholipid molecules, created by the insertion local spacing allows faster and less restricted rotation of the doxyl group. These measurements show that the addition of a NADES to liposome in a buffer results in changes in the liposome membranes. Different NADES have different effects. The effects include for some NADES an increase of the liposome stability. To translate these results to living cells is at this point pure speculation, but at least it is clear that different NADES may interact with different membranes in cells and thus may have a host of functions in the cell. For example one may envisage that the vacuolar membrane on the inside has a sugar-malic acid NADES, because of the acidic nature of the vacuolar liquid, and on the outside a sugar-choline chloride NADES, thus creating a kind of buffer to stabilize the tonoplast potential.

The most important lesson form these experiments is that the addition of NADES to an aqueous liposome suspension, changes the character of the liposomes. This fits our thoughts about the role of NADES in biological systems, NADES is a concept in which complex interactions between various molecules need to be considered to better understand various cellular functions, in which the NADES can be considered as a third liquid phase.

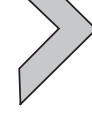

\section{Conclusion}

The aim of this chapter was to summarize and assess the in vitro evidence we accumulated for the existence of NADES in biological systems and to discuss some of their possible functions. NADES were proposed as a third liquid phase in cellular metabolism (Choi et al., 2011). Based on that hypothesis several aspects have been studied. 
A number of flowers have been reported to have much higher levels of flavonoids and anthocyanins than water solubility would enable. These compounds are very well soluble in NADES, containing a certain percentage of water. High levels of these compounds could be found in the extracts of such flowers, but solid phase MAS NMR did not detect the phenolics using a small molecules targeted method. That means that probably in flowers these compounds are bound to large molecules, or form aggregates that behave as high molecular weight compounds and thus remain undetected in the solid phase NMR targeted at small molecules.

NADES may be involved in controlling the water level in plants and even trap water from humid air. NADES components are present in all cells and some are even the major compounds. It is well known that the levels of some NADES components are increased in plants as a defense against freezing and against drought. The use of NADES components in cryopreservation is good evidence for the possible role of NADES against freezing. Further studies in this field maybe important for breeding frost resistant plants and to further optimize cryopreservation of various organisms. Desert plants are obvious subjects for further studies of the role of NADES in resurrection plants as well as studying how plants like cacti can use NADES to hold water or even capture water from the atmosphere when at low temperatures the relative humidity gets very high and hygroscopic sugars based NADES may capture water from the air.

If NADES can be formed in cells, e.g., in vesicles, metabolons or as a layer on cellular membranes, the question is how diffusion would work at the interface between NADES and water in, e.g., the vacuole or the cytosol. An experiment in which a water layer was brought on top of a NADES it was observed that slowly a three-phase system was formed, in which the middle layer was a new NADES with a higher level of water. A dye added to either upper or lower layer diffused upward, but not downward. This shows the complexity of NADES in all kinds of cellular processes.

The stabilizing effects of sugars on membranes and proteins are known already for many years. The experiments we did with NADES showed also clear effects on liposome characteristics, including an increased stability. Assuming that sugars can bind to both membranes and proteins, the NADES open the way to build a whole sheet around the membranes or a protein. Strong reversible hydrogen bonding may result in transiently putting together a series of proteins capable of catalyzing a biosynthetic pathway, i.e., creating a metabolon (Bassard \& Halkier, 2018; see chapter "Metabolons and bio-condensates: The essence of plant plasticity and the 
key elements in development of green production systems" by Møller and Laursen of this issue). The combination of this concept with membranes, leads to vesicles, which creates an environment where non-water-soluble intermediates are dissolved and may be transferred from one enzyme to the next in the sequence of the biosynthesis pathway. The characteristics of an enzyme in a NADES might be quite different from the same enzyme in water, among others because of a much higher concentration of substrate in the NADES liquid phase than in water. To fully address this hypothesis on the central role of NADES in many cellular processes, further experiments are required that can do in situ measurements on the interactions of molecules. Eventually that might revolutionize our views on cell physiology, as well as the physiology of whole organisms.

In this chapter we have brought together the results of a number of separate experiments we did in the past years in which we explored various models for possible roles of NADES in living organisms. Most of the results only give indirect evidence for possible roles, but for the final proof the occurrence, compartmentation and interactions of the NADES molecules in living cells must be mapped, in space and in time, i.e., full characterization of the dynamic system of all molecules in a living cell. Present day omics technologies can characterize all the molecules in an organism and even a cell, but how they interact in a dynamic system and how they are localized on a nanoscale is now the last step into the full understanding of life. A major challenge! A challenge that requires a multidisciplinary approach to be able to unravel all the molecular interactions in the three dimensions of space and the one of time.

\section{Acknowledgments}

We are grateful to Dr. Jieun Shin (Plant Developmental Biology Department, Max Planck Institute, Germany) for providing Arabidopsis plants. We thank KMB Jansen (Mechanics of Materials, Delft University of Technology, the Netherlands) for hydroscopicity tests.

\section{References}

Arakawa, T., \& Timasheff, S. N. (1982). Stabilization of protein structure by sugars. Biochemistry, 21, 6536-6544.

Bartels, D., \& Sunkar, R. (2005). Drought and salt tolerance in plants. Critical Reviews in Plant Sciences, 24, 23-58.

Bassard, J. E., \& Halkier, B. A. (2018). How to prove the existence of metabolons? Phytochemistry Reviews, 17, 211-227.

Buitink, J., \& Leprince, O. (2004). Glass formation in plant anhydrobiotes: Survival in the dry state. Cryobiology, 48, 215-228.

Buitink, J., \& Leprince, O. (2008). Intracellular glasses and seed survival in the dry state. Comptes Rendus Bbiologies, 331, 788-795. 
Choi, Y. H., van Spronsen, J., Dai, Y., Verberne, M., Hollmann, F., Arends, I. W., et al. (2011). Are natural deep eutectic solvents the missing link in understanding cellular metabolism and physiology? Plant Physiology, 156, 1701-1705.

Couch, J. F., Naghski, J., \& Krewson, C. F. (1952). Rutin content of Sophora japonica L. Journal of the American Chemical Society, 74, 424-425.

Crowe, J. H., Crowe, L. M., Carpenter, J. F., \& Aurell Wistrom, C. (1987). Stabilization of dry phospholipid bilayers and proteins by sugars. The Biochemical Journal, 242, 1-10.

Crowe, J. H., Crowe, L. M., Carpenter, J. F., Rudolph, A. S., Wistrom, C. A., Spargo, B. J., et al. (1988). Interactions of sugars with membranes. Biochimica et Biophysica Acta, 947, 367-384.

Crowe, J. H., Hoekstra, F. A., \& Crowe, L. M. (1992). Anhydrobiosis. Annual Review of Physiology, 54, 579-599.

Crowe, J. H., Leslie, S. B., \& Crowe, L. M. (1994). Is vitrification sufficient to preserve liposomes during freeze-drying? Cryobiology, 31, 355-366.

Dai, Y. (2013). Natural deep eutectic solvents and their application in natural products research and development. PhD thesis Leiden University, ISBN:978-90-821172-0-2. Chapter 8.

Dai, Y., Rozema, E., Verpoorte, R., \& Choi, Y. H. (2016). Application of natural deep eutectic solvents to the extraction of anthocyanins from Catharanthus roseus with high extractability and stability replacing conventional organic solvents. Journal of Chromatography. A, 1434, 50-56.

Dai, Y., van Spronsen, J., Witkamp, G. J., Verpoorte, R., \& Choi, Y. H. (2013). Natural deep eutectic solvents as new potential media for green technology. Analytica Chimica Acta, 766, 61-68.

Dai, Y., Verpoorte, R., \& Choi, Y. H. (2014). Natural deep eutectic solvents providing enhanced stability of natural colorants from safflower (Carthamus tinctorius). Food Chemistry, 159, 116-121.

Dai, Y., Witkamp, G. J., Verpoorte, R., \& Choi, Y. H. (2013). Natural deep eutectic solvents as a new extraction media for phenolic metabolites in Carthamus tinctorius L. Analytical Chemistry, 85, 6272-6278.

Dai, Y., Witkamp, G. J., Verpoorte, R., \& Choi, Y. H. (2015). Tailoring properties of natural deep eutectic solvents with water to facilitate their applications. Food Chemistry, 187, 14-19.

Dijksterhuis, J., Nijsse, J., Hoekstra, F. A., \& Golovina, E. A. (2007). High viscosity and anisotropy characterize the cytoplasm of fungal dormant stress-resistant spores. Eukaryotic Cell, 6, 157-170.

El Achkar, T., Fourmentin, S., \& Greige-Gerges, H. (2019). Deep eutectic solvents: An overview on their interactions with water and biochemical compounds. Journal of Molecular Liquids., 288. article number UNSP 111028.

Gutiérrez, M. C., Ferrer, M. L., Yuste, L., Rojo, F., \& del Monte, F. (2010). Bacteria incorporation in deep-eutectic solvents through freeze-drying. Angewandte Chemie (International Ed. in English), 49, 2158-2162.

Kaplan, F., Kopka, J., Sung, D. Y., Zhao, W., Popp, M., Porat, R., et al. (2007). Transcript and metabolite profiling during cold acclimation of Arabidopsis reveals an intricate relationship of cold-regulated gene expression with modifications in metabolite content. The Plant Journal, 50, 967-981.

Knowles, P. F., Marsh, D., \& Rattle, H. W. E. (1976). Magnetic resonance of biomolecules: An introduction to the theory and practice of NMR and ESR in biological systems. United Kingdom: John Wiley \& Sons.

Kovács, Z., Simon-Sarkadi, L., Sovány, C., Kirsch, K., Galiba, G., \& Kocsy, G. (2011). Differential effects of cold acclimation and abscisic acid on free amino acid composition in wheat. Plant Science, 180, 61-68.

Mamajanov, I., Engelhart, A. E., Bean, H. D., \& Hud, N. V. (2010). DNA and RNA in anhydrous media: Duplex, triplex, and G-quadruplex secondary structures in a deep eutectic solvent. Angewandte Chemie (International Ed. in English), 49, 6310-6314. 
Markham, K. R., Gould, K. S., Winefield, C. S., Mitchell, K. A., Bloor, S. J., \& Boase, M. R. (2000). Anthocyanic vacuolar inclusions-their nature and significance in flower colouration. Phytochemistry, 55, 327-336.

Marsh, D. (1981). Electron spin resonance: Spin labels. Molecular Biology, Biochemistry, and Biophysics, 31, 51-142.

Marsh, D., \& Schorn, K. (1998). Correction for anisotropically averaged hypefine splittings and order parameters from pseudopowder electron paramagnetic resonance (EPR) line shapes. An update for slow-motion contributions to lipid spin label spectra from membranes. In L. Berliner (Ed.), Biological Magnetic Resonance (pp. 405-410). New York: Plenum Press.

Mustafa, N. R., de Winter, W., van Iren, F., \& Verpoorte, R. (2011). Initiation, growth and cryopreservation of plant cell suspension cultures. Nature Protocols, 6, 715-742.

Núñez-Pertínez, S., \& Wilks, T. R. (2020). Deep eutectic solvents as media for the prebiotic DNA-templated synthesis of peptides. Frontiers in Chemistry. https://doi.org/10.3389/ fchem.2020.00041.

Pan, Q., Dai, Y., Nuringtyas, T. R., Mustafa, N. R., Schulte, A. E., Verpoorte, R., et al. (2014). Investigation of the chemomarkers correlated with flower colour in different organs of Catharanthus roseus using NMR-based metabolomics. Phytochemical Analysis: $P C A, 25,66-74$.

Pandey, V., Ranjan, S., Deeba, F., Pandey, A. K., Singh, R., Shirke, P. A., et al. (2010). Desiccation-induced physiological and biochemical changes in resurrection plant, Selaginella bryopteris. Journal of Plant Physiology, 167, 1351-1359.

Paniwnyk, L., Beaufoy, E., Lorimer, J. P., \& Mason, T. J. (2001). The extraction of rutin from flower buds of Sophora japonica. Ultrasonics Sonochemistry, 8, 299-301.

Peters, S., Mundree, S. G., Thomson, J. A., Farrant, J. M., \& Keller, F. (2007). Protection mechanisms in the resurrection plant Xerophyta viscosa (Baker): Both sucrose and raffinose family oligosaccharides (RFOs) accumulate in leaves in response to water deficit. Journal of Experimental Botany, 58, 1947-1956.

Pohjala, L., \& Tammela, P. (2012). Aggregating behavior of phenolic compounds-A source of false bioassay results? Molecules, 17, 10774-10790.

Risselada, H. J., \& Marrink, S. J. (2009). Curvature effects on lipid packing and dynamics in liposomes revealed by coarse grained molecular dynamics simulations. Physical Chemistry Chemical Physics, 11, 2056-2067.

Sachse, J. H., King, M. D., \& Marsh, D. J. (1987). ESR determination of lipid translational diffusion coefficients at low spin-label concentrations in biological membranes, using exchange broadening, exchange narrowing, and dipole-dipole interactions. Journal of Magnetic Resonance, 71, 385-404.

Scheidt, H. A., Pampel, A., Nissler, L., Gebhardt, R., \& Huster, D. (2004). Investigation of the membrane localization and distribution of flavonoids by high-resolution magic angle spinning NMR spectroscopy. Biochimica et Biophysica Acta, 1663, 97-107.

Schuurink, R. C., Sedee, N. J., \& Wang, M. (1992). Dormancy of the barley grain is correlated with gibberellic acid responsiveness of the isolated aleurone layer. Plant Physiology, 100, 1834-1839.

Whittaker, A., Martinelli, T., Farrant, J. M., Bochicchio, A., \& Vazzana, C. (2007). Sucrose phosphate synthase activity and the co-ordination of carbon partitioning during sucrose and amino acid accumulation in desiccation-tolerant leaf material of the $\mathrm{C} 4$ resurrection plant Sporobolus stapfianus during dehydration. Journal of Experimental Botany, 58, 3775-3787.

Yancey, P. H., Clark, M. E., Hand, S. C., Bowlus, R. D., \& Somero, G. N. (1982). Living with water stress: Evolution of osmolyte systems. Science (New York, N.Y.), 217, 1214-1222.

Zhao, H., Baker, G. A., \& Holmes, S. (2011). New eutectic ionic liquids for lipase activation and enzymatic preparation of biodiesel. Organic \& Biomolecular Chemistry, 9, 1908-1916. 